Cómo citar este artículo en Chicago: Martínez Romagosa, Pedro. "La cuestión del método en el pragmatismo contemporáneo". Escritos 29, no. 62 (2021): 123-143. doi: http://dx.doi.org/10.18566/escr.v29n62.a08

Fecha de recepción: 26.01 .2021

Fecha de aceptación: 01.03.2021

\title{
La cuestión del método en el pragmatismo contemporáneo
}

\author{
The Issue of Method within Contemporary Pragmatism
}

\author{
Pedro Martínez Romagosa ${ }^{1}$
}

\begin{abstract}
RESUMEN
La discusión acerca de la cuestión del método marca uno de los principales puntos de desacuerdo entre las dos posiciones en disputa en el pragmatismo contemporáneo: el neopragmatismo lingüístico y el pragmatismo de cuño clásico centrado en la experiencia. Aunque no se puede desentender completamente del contexto del debate mencionado en que la falta de comprensión mutua entre las partes bloquea una conversación productiva, considero que la discusión acerca de la cuestión del método puede examinarse por separado. Para ello, en la primera sección, presentaré la propuesta de John Dewey que comprende "la experiencia como método" y evaluaré a continuación el argumento de Richard Rorty a favor de un "pragmatismo sin método". Defenderé que la perspectiva pragmatista "experimental" de la investigación puede responder exitosamente a la crítica rortyana. En la segunda sección, ofrezco una exposición de la propuesta metodológica pragmatista en línea con la perspectiva de Dewey, aunque sugiero resulta compatible también con el neopragmatismo rortyano, y defiendo que ella se comprende mejor como una propuesta metafilosófica acerca de cómo llevar adelante y cuál debe ser la tarea de la investigación filosófica.
\end{abstract}

Palabras clave: Pragmatismo contemporáneo; Experiencia; Lenguaje; Método; Metafilosofía; Richard Rorty; John Dewey.

\section{ABSTRACT}

The issue of method is an area of disagreement and contention between two different perspectives of contemporary pragmatism: linguistic neopragmatism and pragmatism of classical tendency focused on experience. Though the debate within contemporary pragmatism is wider and the context should be considered, I think that the issue of method can be addressed separately. First, the article introduces John Dewey's idea of "experience as method" and considers Richard Rorty's argument in favor of "pragmatism without method". I argue that the "experimental" pragmatist perspective of inquiry can successfully answer to the criticism of the latter. Secondly, the article presents the proposal of pragmatist methodology in line with Dewey's perspective -though I suggest that it is compatible with Rorty's neopragmatism. I argue that this proposal might be better understood as a metaphilosophical one, that is, about what is the purpose of and how to undertake philosophical inquiry.

Keywords: Contemporary Pragmatism; Experience; Language; Method; Metaphilosophy:; Richard Rorty; John Dewey.

1 Instituto de Investigaciones Filosóficas, Universidad de Buenos Aires, Argentina. Sociedad Argentina de Análisis Filosófico - Conicet. Correo electrónico: promagosa@filo.uba.ar 


\section{Introducción}

$\mathrm{E}$ 1 debate en el pragmatismo contemporáneo acerca de la prioridad del lenguaje o la experiencia ha llegado a dividir el movimiento en dos "campos" u "olas", esto es, dos propuestas filosóficas diferentes que se disputan cuál logra articular mejor los insights de la tradición. ${ }^{2}$ El neopragmatismo centrado en el lenguaje considera problemática la apelación de los clásicos a la experiencia no lingüística que pretende cumplir un rol epistemológico justificatorio, pues resulta indistinguible de lo que Wilfrid Sellars denunció como el "mito de lo dado" y no sería un intento fallido de librarse de la imagen tradicional "representacionista" de la mente y "fundacionista" del conocimiento. ${ }^{3}$ En cambio, propone el análisis de las prácticas lingüísticas como una alternativa teórica que no conduce a plantear los problemas de la epistemología tradicional.

Pero el de experiencia es el concepto central de los pragmatistas clásicos, en especial, James y Dewey. Para sus representantes contemporáneos, la reivindicación de un "pragmatismo sin experiencia” amenaza con eviscerar el núcleo conceptual de su propuesta filosófica. ${ }^{4} \mathrm{La}$ apelación a la experiencia no cognitiva, no reflexiva y no discursiva resulta indispensable para la reconstrucción de la investigación que sitúe el conocimiento en su contexto eminentemente práctico. De manera que la defensa "primapragmatista" suele acusar de intelectualista la concepción del lenguaje, los significados o la "práctica social" de los neopragmatistas. ${ }^{5}$

Desafortunadamente, pese al rol que haya tenido la discusión en el resurgimiento del pragmatismo, un creciente consenso identifica un impasse en el debate. ${ }^{6}$ Las acusaciones cruzadas de "fundacionismo" e "intelectualismo" suelen ser desestimadas por "errar el blanco" al comprender mal la posición atacada, lo que podría entenderse como bajo la acusación de caer en la falacia del "hombre de paja”.7 Se ha

2 Steven Levine, Pragmatism, Objectivity, and Experience (Cambridge: Cambridge University Press, 2019), 1-3; Christopher Voparil, "Rorty and Dewey Revisited: Toward a Fruitful Conversation", Transactions of the Charles S. Peirce Society 50, n. 3 (2014): 373-377. Para una presentación del estado de la cuestión, véase David L. Hildebrand, "Introduction", European Journal of Pragmatism and American Philosophy 6, n. ${ }^{\circ} 2$ (2014): 6-13, y Richard Bernstein, The Pragmatic Turn (Malden: Polity Press, 2013), 125-152.

3 Colin Koopman, "Language Is a Form of Experience: Reconciling Classical Pragmatism and Neopragmatism", Transactions of the Charles S. Peirce Society 43, n. 4 (2007): 696-698.

4 Koopman, "Language Is a Form of Experience", 696; Bernstein, The Pragmatic Turn, 128.

5 Bernstein, The Pragmatic Turn, 152; Mark Johnson, "Experiencing Language: What's Missing in Linguistic Pragmatism?", European Journal of Pragmatism and American Philosophy 6, n. 2 (2014): 17; Gregory Fernando Pappas, "John Dewey's Radical Logic: The Function of the Qualitative in Thinking", Transactions of the Charles S. Peirce Society 52, n. 3 (2016): 437. Adoptaré la expresión “primapragmatismo" propuesta por Koopman para referirme al pragmatismo de la experiencia que incluye tanto a los clásicos como a los defensores contemporáneos; Koopman, "Language Is a Form of Experience", 724-725.

6 Koopman, "Language Is a Form of Experience", 696; Bernstein, The Pragmatic Turn, 128-129; Johnson, "Experiencing Language", 14-15 y 19; Voparil, "Rorty and Dewey Revisited", 373-404; David Hildebrand, "Dewey, Rorty, and Brandom: The Challenges of Linguistic Neopragmatism”, en The Oxford Handbook of Dewey, ed. por Steven Fesmire (Oxford University Press, 2019), 98-130.

7 éase, por ejemplo, Robert Brandom, Perspectives on Pragmatism: Classical, Recent, and Contemporary (Harvard University Press, 2011), 123, y Gregory Pappas, “What Difference Can 'Experience' Make to Pragmatism?”, European Journal of Pragmatism and American Philosophy 6, n. ${ }^{\circ} 2$ (2014): 201. 
denunciado que se construye la distinción entre experiencia y lenguaje como un falso dilema, una dicotomía de la clase que los pragmatistas clásicos pretendían haber superado. ${ }^{8} \mathrm{El}$ foco impuesto en la oposición lenguaje/experiencia suele desviar el debate a discusiones poco productivas, como la relación entre experiencia o pensamiento y lenguaje.

En este panorama, la toma de posición acerca de la cuestión del método marca uno de los principales puntos de desacuerdo en el movimiento, pues, para los pragmatistas clásicos, la experiencia no solo se encontraba a la base de sus respectivas propuestas filosóficas sustantivas (la metafísica naturalista, la "lógica" o teoría de la investigación de Dewey o la teoría de la verdad de James), sino que también ofrecía un método general de investigación que encontraban paradigmáticamente aplicado en la práctica de investigación de las ciencias naturales, pero reivindicaban su adopción para la reflexión filosófica, mientras que la posición neopragmatista, en especial, la de Rorty, insistió no solo en abandonar el concepto de experiencia y su sustitución por "lenguaje" (junto con el intento de redescripción de la metafísica, la epistemología o una teoría explicativa de la verdad), sino que también cuestionó el compromiso teórico de los clásicos con la idea de "método".

Sin embargo, aunque no puede desentenderse del contexto del debate más amplio acerca de la prioridad de la experiencia o el lenguaje, considero que los argumentos que motivan el desacuerdo en el pragmatismo sobre la cuestión del método pueden ser examinados por su cuenta, pues el propio Rorty identificó como dos diferencias distintas entre su posición y la de los clásicos, y planteó sus razones separadamente respecto de ambas cuestiones. Además, el desacuerdo sobre la posición metodológica entre ambas partes resulta, como intentaré mostrar, menos profundo, a pesar de que, curiosamente, ha recibido menor atención en la discusión en comparación con la cuestión de la relación entre experiencia y lenguaje o cómo entender ambos conceptos.

Abordaré, entonces, la cuestión del método en el pragmatismo contemporáneo y en la sección dos examinaré críticamente el trasfondo teórico de la discusión. Para plantear, primero, las posiciones enfrentadas, presentaré la comprensión de Dewey "la experiencia como método" y la crítica de Rorty para un "pragmatismo sin método". Y, segundo, evaluaré los argumentos que motivan el desacuerdo entre ambas partes. Defenderé que entre el "anarquismo" metodológico que recomienda la crítica rortyana y el realismo epistemológico robusto que se compromete con una concepción universalista del método, hay espacio para una posición intermedia, pues del rechazo de la segunda posición no se sigue forzosamente la primera.

En la sección tres, propongo una exposición de la metodología pragmatista en línea con la perspectiva de Dewey defendida en la primera sección, capaz de responder exitosamente a la crítica rortyana. Enfatizaré el carácter metafilosófico de la propuesta, y sugeriré cómo puede ser aplicada a la investigación filosófica contemporánea.

8 Bernstein, The Pragmatic Turn, 128-129; Johnson, "Experiencing Language”, 14-15.

9 Richard Rorty, "Between Hegel and Darwin", en Truth and Progress: Philosophical Papers (Cambridge, Cambridge University Press, 1998), 3:290-306; y Richard Rorty, Philosophy and Social Hope (Londres, Penguin Books, 1999$), 35$. 


\section{Marco teórico}

A modo de aclaración preliminar, cabe notar que, aunque esta sección se centra en la exposición crítica de posiciones centrales en el desarrollo del pragmatismo contemporáneo, mi principal interés no es histórico sino filosófico. El propósito de las reconstrucciones ofrecidas consiste en permitir evaluar cómo impactan las distintas posiciones y su desacuerdo sobre la discusión actual.

\section{La experiencia como método en el pragmatismo de Dewey}

El pragmatismo de Dewey, que denominó "naturalismo experimental" o "humanista", aunque luego prefirió "naturalismo cultural", se desarrolla en dos direcciones. ${ }^{10}$ Por un lado, ofrece un diagnóstico crítico del proyecto filosófico moderno de la "industria epistemológica" centrada en la "imagen del espectador" de la mente, el conocimiento y el lenguaje heredada del pensamiento antiguo griego. ${ }^{11}$ Por otro, propone realizar una reconstrucción de la filosofía como crítica cultural para que deje de ocuparse de "los problemas de la filosofía" para hacerlo de los problemas de los seres humanos. ${ }^{12}$ Dewey comprendía que el despliegue de su propuesta en ambas direcciones se encontraba integrado en un mismo movimiento de investigación que identificaba con la aplicación del "método experimental" en filosofía y tenía como centro la recuperación del concepto de experiencia. ${ }^{13}$

Desde una perspectiva historicista legada de Hegel, Dewey propuso comprender las teorías y sistemas de la historia de la filosofía en el contexto más amplio de la cultura como reflejo de las necesidades, los conflictos y los problemas sociales. ${ }^{14}$ De acuerdo con su análisis histórico intelectual de la experiencia, la formulación de los problemas metafísicos y epistemológicos de la filosofía moderna dependía de presupuestos teóricos arraigados en la tradición intelectual europea en una serie de dualismos, cuyo origen se remontaba al pensamiento antiguo griego (sujeto-objeto, experiencia-naturaleza, hechos-valores). Pero la adopción de los presupuestos dualistas en cuestión dependía de condiciones históricas, sociales y culturales, precientíficas y preindustriales, que diferían radicalmente, a juicio de Dewey, de las actuales. De manera que, de la contingencia de las condiciones intelectuales de las que depende la adopción de los presupuestos filosóficos tradicionales, se sigue su carácter optativo. Además, al encontrarse disponible un modelo alternativo de la experiencia y el conocimiento, y quedar en evidencia el carácter anacrónico

10 John Dewey, Experience and Nature (Nueva York: Dover Publications, 1958); John Dewey, "Experience, Knowledge and Value: A Rejoinder", en The Philosophy of John Dewey, ed. por Paul Schilpp y Lewis Hahn (Chicago: Open Court, 1989), 517-608; David Hildebrand, “John Dewey", Stanford University, acceso el 7 de abril de 2021, https://plato. stanford.edu/archives/win2018/entries/dewey/.

11 Dewey, Experience and Nature, 18-23, y Dewey, "Experience, Knowledge and Value”, 522-524. Para una reconstrucción sistemática de la estrategia de diagnóstico, véase Angel Faerna, "Pragmatismo conceptualista: La teoría del conocimiento de C. I. Lewis" (tesis doctoral, Universidad Complutense de Madrid, 2002), 111-127, acceso el 7 de abril de 2021, http://eprints.ucm.es/2310/.

12 Dewey, Experience and Nature, 37-39 y 394-437; John Dewey y Sidney Ratner, The Later Works of John Dewey (Carbondale: Southern Illinois University Press, 1985), 6:17-21; Richard Bernstein, Filosofía y democracia: John Dewey (Barcelona: Herder, 2011), 48-60 y 70.

13 Rorty critica la unidad del proyecto de Dewey en Richard Rorty, "Dewey's Metaphysics”, en Consequences of Pragmatism (Minneapolis: University of Minnesota Press, 1982), 72-89. Para una respuesta, véase Bernstein, The Pragmatic Turn, 144-152; Hildebrand, "Dewey, Rorty, and Brandom”, 99-120.

14 Dewey, Experience and Nature, 36-37; Dewey, “Context and Thought”, 17; Dewey, "Experience, Knowledge and Value”, 522. 
de los dualismos, el pragmatista clásico defiende que los problemas filosóficos tradicionales resultan ociosos, además de anacrónicos, y deben ser lisa y llanamente abandonados. ${ }^{15}$

El pragmatismo, además, proponía Dewey, lleva adelante una "reconstrucción de la filosofía" desde la perspectiva naturalista experimental. Por un lado, adhirió a la comprensión hegeliana de la experiencia como actividad que se despliega histórica, social y culturalmente: la articulación conceptual tiene lugar a partir de la resolución de conflictos o tensiones internas que son "superados" al ser incorporados los elementos en oposición a totalidades más amplias que las incluyen como distinciones funcionales. ${ }^{16}$ Pero, motivado por el impacto cultural de la obra de Darwin, se apropió y transformó la noción hegeliana en clave naturalista. ${ }^{17}$ Comprendió la experiencia primariamente respecto de la conducta del organismo en adaptación al medio físico y social, y en el caso de los seres humanos, cultural, ${ }^{18}$ e identificó en la nueva psicología funcionalista inspirada en la biología una comprensión alternativa a la imagen mentalista y atomista tradicional, hasta el punto de que su formulación seminal fue elaborada a propósito de la redescripción del concepto de arco reflejo en psicología. ${ }^{19}$

El otro factor clave en la historia intelectual para la nueva concepción de la experiencia fue, para Dewey, el desarrollo del método experimental. El pragmatista clásico consideraba que en la práctica de investigación de las ciencias naturales se encontraba implícito un "método" y una concepción del conocimiento alternativos al de la tradición intelectual europea heredadas del pensamiento antiguo griego. ${ }^{20} \mathrm{La}$ imagen contemplativa del "espectador", por un lado, reflejaba la división de clase de la sociedad antigua griega entre los esclavos a cargo del trabajo corporal y "hombres libres" dedicados a la dirección política, militar y religiosa, de manera que la separación y el privilegio del pensamiento y la teoría en desmedro de la práctica y la acción reflejaban el prejuicio social de clase. ${ }^{21}$ Y por otro, la imagen respondía a las condiciones limitadas del conocimiento científico, en las que el modelo del conocimiento era la aprehensión intelectual de un objeto inmediatamente presente a la mente que involucraba certeza. ${ }^{22}$

Por el contrario, la concepción experimental ponía a la práctica de experimentación en el corazón del

15 Dewey, Experience and Nature, 18, 21-23.

16 Faerna, Introducción a la teoría pragmatista del conocimiento, 174-187; Bernstein, The Pragmatic Turn, 144-45; Hildebrand, "John Dewey"; Levine, Pragmatism, Objectivity, and Experience, 10-15.

17 Véase especialmente John Dewey, The Influence of Darwin on Philosophy and other essays (Nueva York: Prometheus Books, 1997), 1-19.

18 La interacción humana está mediada por la comunicación lingüística; cf. Dewey, Experience and Nature, 166 y ss. Como enseña la antropología, los seres humanos son "organismos aculturados"; cf. Dewey, "Experience, Knowledge and Value", 530.

19 Desde la perspectiva de la conducta del organismo en adaptación al medio, "estímulo" y "respuesta" son comprendidos como distinciones funcionales en la unidad del comportamiento. El estímulo nunca es un átomo de sensación aislado en relación externa con la respuesta. La conducta supone un conjunto de hábitos establecidos, una "coordinación sensorio-motriz", de manera que, cuando la respuesta es indeterminada, el estímulo también lo es. La sensación no es primariamente un contenido de información, sino un "estímulo para la acción”, de la conducta habitual o en el caso de que se trate de un conflicto entre hábitos para establecer un nuevo modo de conducta; John Dewey, La miseria de la epistemología, ed. por Ángel Faerna (Madrid: Biblioteca Nueva, 2000), 99-112.

20 Dewey, Experience and Nature, 1-39; Dewey, "Experience, Knowledge and Value", 526-530; Bernstein, Filosofía y democracia, 67-68.

21 John Dewey, La busca de la certeza (México: Fondo de Cultura Económica, 1952), 4-21.

22 Dewey, La busca de la certeza, 15-22. 
conocimiento, ${ }^{23}$ pues la actividad de conocer tiene lugar en una situación de indeterminación, en que, debido a algún cambio en la relación con el medio, se produce un conflicto o tensión entre los modos de conducta establecidos y medios de acción disponibles de manera que resulta incierto cómo actuar. De manera que la investigación es comprendida respecto de la transformación controlada de la situación hacia su determinación, en que se establece un nuevo modo de acción que involucra una nueva relación de equilibrio con el medio. ${ }^{24}$

Dewey, entonces, desarrolló su "teoría de la investigación" a partir de la concepción experimental del conocimiento que encontraba implícita paradigmáticamente en la práctica de investigación de las ciencias naturales, pero que consideraba aplicable a toda práctica inteligente humana (la moral, la política, el arte, etc.). De manera que su comprensión general de la investigación ofrecía un método que propuso aplicar, en especial, a la reflexión filosófica, y llegó, incluso, a identificar como la contribución principal de su propuesta pragmatista.

\section{El argumento de Rorty por un "pragmatismo sin método"}

Rorty encuentra una tensión en el pragmatismo clásico en la oscilación por parte de Peirce, James y Dewey entre el intento de elevar las distintas áreas de la cultura al estatus epistemológico especial de las ciencias naturales y el de "nivelar para abajo" a la ciencia para equipararla con la política, la moral y demás prácticas humanas, ${ }^{25}$ pues considera que en la escena pública la principal función social y cultural del movimiento fue la crítica de las creencias, prácticas e instituciones heredadas de la tradición, en particular, en una nación en que la cultura religiosa permeaba la vida pública. Y, en su intento de romper con la influencia de la tradición y reemplazar los antiguos códigos morales mediante la "actitud experimental”, el pragmatismo de los clásicos reivindicó el método del científico experimental como modelo para el resto de la cultura. ${ }^{26}$ Sin embargo, en la comunidad filosófica, los pragmatistas clásicos se diferenciaron del naturalismo ortodoxo, el cientificismo y el empirismo clásico, y se los conocía mejor como "holistas", que se oponían al esencialismo, el atomismo y representacionismo dominante. ${ }^{27}$

Para Rorty, en vez de pretender elevar las demás áreas de la cultura al estatus epistemológico privilegiado de la ciencia mediante la aplicación del método, la vertiente holista del pragmatismo parte de reconocer que el conocimiento es una práctica social, y propone establecer una continuidad entre las prácticas sociales (política, moral, arte, etc.). Rorty consideraba el compromiso de los clásicos con la "primacía del método" un legado desafortunado de la tradición intelectual, como el proyecto de una teoría del conocimiento, ${ }^{28}$ pues, además de generar un compromiso innecesario con el positivismo cientificista,

23 Dewey, La busca de la certeza, 32-33.

24 Dewey, La miseria de la epistemología, 117.

25 Richard Rorty, "Pragmatism Without Method", en Philosophical Papers (Cambridge: Cambridge University Press, 1991), 1:63.

26 Rorty, "Pragmatism Without Method", 1:63.

27 Rorty, "Pragmatism Without Method", 1:64.

28 Richard Rorty, "Method, Social Science and Social Hope", en Consequences of Pragmatism (Minneapolis: University of Minnesota Press, 1982), 191-95."page":"1912-210"',publisher":"University of Minnesota Press'”,publisher-place":" Minneapolis"'”title":"Method, Social Science and Social Hope'”'author":[\{“family":”Rorty','given":”Richard”\}],"issued ":\{“date-parts":[[“1982”]]\}\},"locator":"191-195”\}],"schema”:"https://github.com/citation-style-language/schema/raw/ master/csl-citation.json”\} 
involucraría una forma de autoritarismo ético-epistemológico a contramano de la propuesta pragmatista, puesto que conduciría a comprender el método como una especie de autoridad no humana, independiente de la práctica social y del acuerdo de la comunidad a la que los participantes deberían someterse para comportarse adecuadamente, éticamente o en calidad de investigadores. ${ }^{29}$

Para presentar, y luego poder evaluar, la crítica de Rorty al pragmatismo del método, considero conveniente distinguir las objeciones tempranas del neopragmatista de su crítica posterior, ya que, en la primera formulación, Rorty se dirige directamente a los clásicos, en particular, a Dewey, ${ }^{30}$ mientras que posteriormente sus razones para dudar acerca del método se despliegan a partir de su discusión con el discípulo de Dewey: Sidney Hook. Como intentaré mostrar, a mi juicio, Rorty logra desarrollar de forma más acabada su argumento en contra del pragmatismo del método en la formulación posterior.

La crítica de Rorty a la comprensión de Dewey del método parte de reformular una objeción realizada originalmente a su posición idealista temprana, pues, en su periodo anterior, Dewey pretendía haber encontrado en la práctica del psicólogo experimental el método de la filosofía y la ciencia en su conjunto, y consideraba que la psicología trataba "con la experiencia en su totalidad absoluta" sin separar o aislar ningún aspecto de ella. Pero críticos contemporáneos como Shadworth Hodgson acusaron, entonces, al joven Dewey de no haber ofrecido una caracterización positiva del "método" más allá de dar una descripción negativa acerca de los errores habituales de los psicólogos y el empirismo clásico.

Rorty sostiene que puede encontrarse la misma confusión denunciada por Hodgson en el periodo de madurez de la obra de Dewey, en su propuesta de adopción del método de la ciencia experimental para la reconstrucción de la metafísica naturalista, puesto que el clásico da a entender que, a partir de la "revolución científica", se encontraba disponible un nuevo método de investigación que había mostrado su éxito en las ciencias naturales, cuya aplicación recomendaba para la investigación en ciencias sociales, moral, política, arte, etc. Sin embargo, tal como objetara Hodgson en su juventud, Dewey nunca habría llegado a ofrecer una caracterización del método como tal. ${ }^{31}$

La objeción del neopragmatista a Dewey, entonces, reitera la crítica de Hodgson y denuncia una ambigüedad en la comprensión del "método" que considera fatal, pues el uso del término por parte del clásico, sostiene Rorty, oscila entre dos extremos: ${ }^{32}$ por un lado, recomienda la adopción de la actitud "experimental" de la práctica científica a las demás áreas de la cultura; pero, por otro, pretende proponer

29 Rorty, "Pragmatism Without Method", 64.

30 Rorty, "Dewey's Metaphysics", 77-80; Richard Rorty, "Introduction”, en The Later Works of John Dewey, ed. por Jo Ann Boydston (Carbondale: Southern Illinois University Press, 1986), 8:IX-XVIII."page":"72-89','publisher":"University

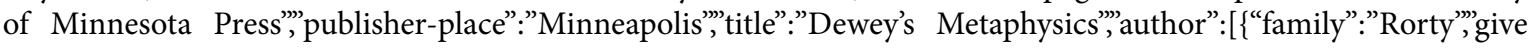
n":"Richard”\}],"issued”:\{“date-parts”:[[“1982”]]\}\}\},\{“id”:32,"uris”:[“http://zotero.org/users/local/AFtThozY/items/ TVG57K9B”],"uri”:[“http://zotero.org/users/local/AFtThozY/items/TVG57K9B”],"itemData”:\{“id”:32,"type”:"chapter "','container-title":"'The Later Works of John Dewey, Volume 8, 1925 - 1953: 1933, Essays and How We Think, Revised

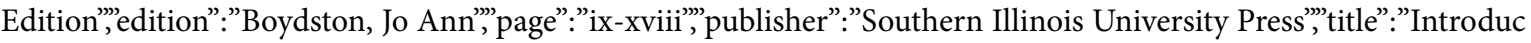

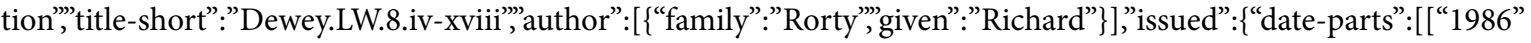
]]\}\}\}],"schema":"https://github.com/citation-style-language/schema/raw/master/csl-citation.json"\}

31 Rorty, "Dewey's Metaphysics", 79; Rorty “Introduction”, XIV.

32 Rorty, “Introduction”, XIII; Richard Rorty, “Response to James Gouinlock”, en Rorty \& Pragmatism: The Philosopher Responds to His Critics, ed. por Herman Saatkamp (Nashville: Vanderbilt University Press, 1995), 92-93. 
una suerte de técnica específica para resolver un problema particular. De manera que, en el primer extremo, el "método" no sería más que la actitud "experimental", crítica, falibilista, antidogmática, consistente en un conjunto de hábitos o "virtudes" intelectuales de la práctica social (de investigación), que, según Rorty, podría resumirse en el eslogan de Peirce de "no bloquear el camino de la investigación", mientras que, en el otro extremo, el método consistiría en un procedimiento concreto, capaz de explicitarse en una serie de reglas o definirse en una lista de instrucciones (como una receta o un algoritmo) que indicaría qué hacer para resolver un problema particular.

De acuerdo con Rorty, la caracterización de Dewey sobre el método es inestable y resulta inviable, pues no cabe adoptar una posición intermedia que reúna ambos extremos. ${ }^{33}$ Por supuesto, para fines específicos, en un contexto particular, puede ofrecerse una descripción adecuada de una "técnica" o dar instrucciones acerca de cómo seguir un procedimiento concreto, pero una práctica social como tal no puede definirse mediante una serie de reglas explícitas. Desde la perspectiva wittgensteiniana de Rorty, no se puede determinar el significado lingüístico de una expresión mediante una definición o "explicación ostensiva". ${ }^{34}$ En su aprendizaje, pueden señalarse ejemplos como parte del adiestramiento, pero ello supone la participación de los hablantes en prácticas lingüísticas compartidas en una comunidad. Análogamente, Kuhn comprende el entrenamiento y la formación en la "ciencia normal" del investigador mediante "ejemplares", pero el consenso de la comunidad científica que rige la práctica de una disciplina en un periodo histórico determinado no puede definirse o explicitarse mediante una serie de reglas, lo que llamó "paradigmas". ${ }^{35}$

Rorty concluye, entonces, que Dewey debe enfrentar un dilema genuino. O bien su discurso acerca del "método" refiere algo general y poco específico como la actitud experimental, o bien las virtudes de la práctica científica, capaz de adoptarse al enfrentar cualquier problema del amplio ámbito de la conducta inteligente humana, desde la práctica de la democracia, pasando por la deliberación moral y las investigaciones científicas, pero que, en sentido estricto, no ofrece ninguna indicación metodológica acerca de qué hacer en una investigación particular. O bien, su propuesta del "método" ofrece un procedimiento o conjunto de indicaciones concretas acerca de cómo resolver un problema particular: una "técnica" suficientemente específica capaz de ser evaluada en comparación con otros "métodos" alternativos disponibles; sin embargo, la propuesta metodológica no puede ofrecer ambas cosas a la vez. Además, Rorty agrega un punto aún más radical a su denuncia de ambigüedad contra la comprensión del método de Dewey, puesto que llega a sostener que encuentra una tensión entre la dimensión descriptiva y la dimensión normativa o prescriptiva de la propuesta del clásico. ${ }^{36}$ En su periodo temprano, Dewey presenta su concepción de la enseñanza, en calidad de especialista, como valorativamente neutral, pero pretendería imponerla con las mismas credenciales en el debate público en educación como instrumento para el cambio social progresivo. ${ }^{37}$

33 Rorty, "Introduction”, XIII; Rorty, "Response to Gouinlock”, 92-93.

34 Richard Rorty, Philosophy as Cultural Politics: Philosophical Papers (Cambridge, University Press, 2007), 117; Ludwig Wittgenstein, Investigaciones filosóficas (Madrid: Gredos, 2009), 21 y 41-43.

35 Thomas Kuhn, La estructura de las revoluciones científicas (Buenos Aires, Fondo de Cultura Económica, 1988), 68-90.

36 Rorty, "Introduction”, XII; Rorty, “Dewey’s Metaphysics”, 74, 78 y 80.

37 Rorty, "Introduction", XI. 
Análogamente, en su obra de madurez, Experience \& Nature, Dewey sostiene que la adopción del "método experimental" en filosofía permite denunciar y evitar la "falacia filosófica”. Las respuestas históricas metafísicas y epistemológicas a los problemas centrales de la disciplina ignoraban el contexto práctico de la investigación en que se instituye y aplica algún concepto o distinción, convierten una distinción funcional en una realidad antecedente (hipóstasis), y así pretenden ofrecer una justificación metafísica última de una práctica social (las normas o los valores encarnados en ella). ${ }^{38}$

Pero mediante la misma aplicación del "método experimental” Dewey pretendería ofrecer una "metafísica de la experiencia": una descripción de los rasgos generales de toda existencia independiente de su carácter subjetivo u objetivo, físico o mental, fáctico-valorativo, natural o cultural. ${ }^{39}$ Rorty considera que, como en su periodo de juventud, Dewey no se contentaba con sostener que ofrecía un mejor análisis de la experiencia que el empirista clásico o el idealista, sino que también brindaba un acceso directo a la experiencia tal como es en sí misma. De manera que, aunque en su periodo de madurez abandonara la perspectiva metafísica tradicional a priori “sub specie aeternitatis", Dewey aún pretendería preservar una perspectiva neutral para la investigación filosófica. ${ }^{40}$

Finalmente, Rorty reformuló posteriormente sus razones en contra del "pragmatismo del método" una vez desarrollada su posición filosófica de madurez, y publicada buena parte de su obra, ocasión en la que no discute directamente ya con Dewey, sino que también su argumento se despliega a partir de la conversación con Hook.

El discípulo de Dewey sigue a su maestro al caracterizar el pragmatismo como una variedad de naturalismo comprometido no solo con "las generalizaciones amplias" establecidas por las investigaciones científicas exitosas, sino también con el método de conocimiento utilizado en ellas. ${ }^{41}$ Pero, según Rorty, Hook cedería a la tentación que acecha al naturalista ortodoxo representacionista de pretender acceder mediante el método a una base neutral de evidencia que permita dirimir definitiva y concluyentemente la discusión con el antinaturalista, pues reformula el contraste que establecía Dewey entre la actitud contemplativa de los sacerdotes y la actitud práctica de los artesanos respecto de la distinción entre los enunciados que tienen contenido cognitivo y los que no. ${ }^{42}$ De manera que distingue los enunciados de la ciencia, que tienen contenido empírico, de los de la religión, que no lo tienen.

Para Rorty, el pragmatismo "del método" se aparta en este punto del antirrepresentacionismo "holista”, como el de James en su comprensión de la creencia religiosa, y considera que, tras las críticas contemporáneas a los "dogmas" del empirismo, ya no resulta plausible trazar una distinción tajante entre la teoría y la evidencia empírica. ${ }^{43}$ Para Rorty, la crítica de Quine a la distinción analítico-sintético permitió mostrar el fracaso del intento de separar los enunciados que se aceptan en virtud de "los hechos" de las verdades "analíticas", que se aceptan como resultado de los significados. Por su parte, Davidson desarrolló aún más

38 Rorty, "Dewey"s Metaphysics", 74.

39 Rorty, "Dewey's Metaphysics", 74 y 80.

40 Rorty, "Dewey's Metaphysics", 80.

41 Sidney Hook, The quest for Being (Nueva York: Macmillan, 1961), 185; Rorty, “Pragmatism Without Method”, 65.1961

42 Rorty, "Pragmatism Without Method", 66; Hook, The quest for Being, 180.

43 Rorty, Objectivity, Relativism, and Truth, 113-25. 
la crítica al empirismo en su rechazo al dualismo esquema/contenido, ya que, desde la perspectiva del intérprete, no se pueden determinar independientemente los significados de las expresiones, tal como son usadas en las oraciones de los hablantes, y las creencias que mantienen acerca del mundo. ${ }^{44}$

Hook, además, caracteriza su compromiso pragmatista con el método experimental respecto de la concepción de la filosofía clásica de la ciencia de la "lógica de la investigación"; según Rorty, en un intento de ganarse la alianza con los empiristas lógicos. De acuerdo con la posición de Hook, "hay un solo método confiable para alcanzar la verdad acerca de la naturaleza de las cosas de todo tiempo y todo lugar" ${ }^{45}$ Y mantiene que "lógica del método científico" ofrece principios de validez universal que no dependen de factores históricos, sino que justifican la aceptación y el rechazo racional de hipótesis científicas independiente del contexto. ${ }^{46}$

Como se recordará, según la distinción clásica entre contexto de descubrimiento y contexto de justificación, la "concepción heredada" en filosofía de la ciencia reconocía que los científicos podrían apelar a cualquier recurso que dispusieran para inspirarse a la hora de proponer problemas e hipótesis científicas, ${ }^{47}$ ya que consideraban que no había un método de descubrimiento: un procedimiento, una serie de patrones o un algoritmo que indicara a los investigadores los pasos a seguir para llegar a plantear problemas relevantes, preguntas interesantes, o formular hipótesis fecundas. En cambio, entendían que la objetividad del conocimiento científico dependía de la aplicación del método de contrastación experimental: los procedimientos paradigmáticamente experimentales que realizaban los investigadores para confirmar o refutar las hipótesis científicas. ${ }^{48}$ Inspirados en los desarrollos recientes de la lógica matemática contemporánea, orientaron el estudio filosófico de la ciencia hacia un análisis lógico-semántico del conocimiento científico, y se dedicaron a tareas como la reconstrucción racional de la estructura lógica de las teorías científicas, la elucidación del esquema argumentativo involucrado en la explicación científica o el análisis de las reglas de inferencia de la justificación de los enunciados científicos. ${ }^{49}$

Así pues, los empiristas lógicos (y otros autores dentro de la "concepción heredada" como Popper) abordaron al menos dos cuestiones distintas mediante el análisis del "conocimiento científico" y la elucidación del "método experimental". Por un lado, el "problema de la demarcación" que involucraba la tarea de establecer condiciones necesarias y suficientes para distinguir los enunciados de la ciencia de los no científicos, ${ }^{50}$ y por otro, el problema de la confirmación o, en general, del análisis de la "lógica" de la

44 La tarea de interpretación debe dar cuenta simultáneamente de los significados de las emisiones y de las creencias de los hablantes; Rorty, Objectivity, Relativism, and Truth, 136-139.

45 Hook, The quest for Being, 185.

46 Hook, The quest for Being, 185; Rorty, "Pragmatism Without Method", 65.

47 La distinción es originalmente establecida en Hans Reichenbach, Experience and Prediction (Chicago, University of Chicago Press, 1938), 6-7, pero se extendió en la concepción clásica de la filosofía de la ciencia. Véase, por ejemplo, Karl Popper, La lógica de la investigación científica (Madrid: Tecnos, 1980), 30-32; Carl Hempel, Filosofía de la ciencia natural (Madrid: Alianza, 1984), 20-34.

48 Hempel, Filosofía de la ciencia natural, 34.

49 Para una exposición sistemática, véase Harold Brown, "Filosofía de la ciencia del empirismo lógico", en La nueva filosofía de la ciencia (Madrid: Tecnos, 1983), 17-102; Alfredo Marcos, Ciencia y acción: Una filosofía práctica de la ciencia (México: Fondo de Cultura Económica, 2010).

50 Popper, "Lógica de la investigación", 33-38; Hempel, "Filosofía de la ciencia natural", 53-56. 
justificación del conocimiento científico, ${ }^{51}$ cuya tarea consistía en elucidar la estructura de razonamiento involucrada en la contrastación experimental que justificaba la aceptación o rechazo racional de los enunciados de las hipótesis científicas. ${ }^{52}$

Ahora bien, Rorty considera que los desarrollos filosóficos más importantes de la segunda mitad de siglo XX han mostrado el fracaso del proyecto de elucidación o "reconstrucción racional" del método científico. En filosofía de la ciencia, tras los sucesivos fracasos de los intentos de establecer un criterio estricto de demarcación entre enunciados científicos y no científicos, el problema fue abandonado. ${ }^{53}$ La comunidad alcanzó un consenso generalizado sobre el asunto al considerar que no resulta plausible identificar condiciones necesarias y suficientes para distinguir los enunciados científicos de los no científicos. En efecto, los propios empiristas debilitaron sucesivamente las propuestas y terminaron por abandonar la empresa. ${ }^{54}$

A su vez, en virtud de las críticas contra el método de Paul Feyerabend y el éxito de la obra de Thomas Kuhn, la filosofía de la ciencia abandonó la idea de "método" en el sentido de un canon de justificación racional o conjunto de principios epistemológicos ahistóricos y universales. ${ }^{55}$ Desde la perspectiva de la "nueva filosofía de la ciencia", el "aparato de la lógica de la confirmación" se volvió un obstáculo para comprender cómo opera la investigación científica efectiva; ${ }^{56}$ lo que cuenta como "científico" en un área de investigación y lo que sea una buena razón para el cambio de teoría dependen del consenso amplio de la comunidad en la práctica científica en un periodo determinado.

Además, Rorty traza una analogía entre el giro pragmático en filosofía de la ciencia y el diagnóstico de Dewey en filosofía moral y su propuesta experimentalista en ética. Así como la nueva filosofía de la ciencia considera que poner el foco exclusivamente en el análisis lógico semántico de la justificación se volvió un obstáculo para comprender la práctica efectiva de investigación, Dewey estima que la formulación moral tradicional de los dilemas éticos, respecto de un conflicto entre enfoques teóricos centrados exclusivamente en la obediencia a la ley, la búsqueda de la felicidad o la virtud, impiden la comprensión de la práctica efectiva de la deliberación moral. Filósofos como Kuhn consideran que solo en atención al desarrollo históricamente situado de la ciencia, consenso en la comunidad en la práctica efectiva de la ciencia, se puede contar con criterios normativos acerca de qué hipótesis cuentan como "científicas" y cuáles no, y qué cuenta como una buena razón para el cambio teórico, mientras que la tesis

51 Puede identificarse una tercera cuestión a propósito de la distinción ulterior entre contexto de justificación y de aplicación; cf. Gregorio Klimovsky, Las desventuras del conocimiento científico: Una introducción la epistemología (Buenos Aires: A-Z, 1997), 29). La posición que se impuso en la "concepción heredada" fue la de la neutralidad valorativa que recogía el "ideal de la ciencia libre de valores”. La aplicación de los cánones de justificación racional del conocimiento científico excluía la intervención de valores no epistémicos en el contexto de justificación.

52 Véase, por ejemplo, Hempel, Filosofía de la ciencia natural, 38-75; Popper, "Lógica de la investigación”, 32-33, 75-88; Brown, "La filosofía de la ciencia del empirismo lógico", 29-44.

53 Rorty, "Pragmatism Without Method", 67-68.

54 Carl Hempel, "Problemas y cambios en el criterio empirista de significado", en El positivismo lógico, ed. por Alfred Ayer (Madrid: Fondo de Cultura Económica, 1978), 115-36.

55 Paul Feyerabend, Contra el método: Esquema de una teoría anarquista del conocimiento (Buenos Aires: Hyspamerica, 1984); Kuhn, La estructura de las revoluciones científicas.

56 Rorty, "Pragmatism Without Method", 68. 
central de la ética experimentalista de Dewey sostiene que en la deliberación moral los medios para la conducta y los fines se determinan recíprocamente. No se puede separar en la evaluación de la conducta adecuada, en una situación de conflicto particular, la deliberación acerca de los medios de la deliberación acerca de los fines.

De manera que, aunque Rorty no lo haga explícitamente, la crítica contra la posición cientificista de Hook puede comprenderse, a mi juicio, respecto de la denuncia de Dewey a la "falacia filosófica", pues, en efecto, el error de la posición positivista consistiría en ignorar el carácter contextual y el estatus contingente de las normas que ofician de estándares de corrección en la práctica de investigación de las ciencias naturales. De manera que se llega a concebir los "cánones de justificación" locales de la ciencia, en áreas particulares de investigación durante un periodo determinado, como el contenido esencial del "conocimiento" y el "método científico" universal, y se convierten los productos de una práctica social, contingente y contextual en una realidad antecedente (universal y necesaria). De modo tal que se pretende ofrecer una justificación metafísica última de un conjunto de criterios normativos de una práctica social, las normas y los valores de la práctica de investigación científica. Así, el "método científico" se convierte en una especie de autoridad última, de carácter no humano, que resulta independiente de la práctica social de la investigación y el consenso de la comunidad, ${ }^{57}$ y a la que los participantes de la investigación deben someterse y obedecer para comportarse científicamente.

\section{Evaluación y conclusión preliminar}

Como sugerí arriba, considero que la crítica tardía de Rorty logra desarrollar más acabadamente sus razones en contra de la primacía pragmatista del método. En efecto, en la medida en que la posición de Hook se compromete con una concepción universalista, que pretende ofrecer principios ahistóricos de aceptación racional de hipótesis científicas, el argumento de Rorty resulta efectivo.

El cambio de interlocutor favorece, a mi juicio, la plausibilidad de la articulación de una crítica pragmatista en contra del compromiso cientificista. De acuerdo con la reconstrucción propuesta, en su versión posterior el argumento rortyano se fortalece considerablemente, pues identifica un conflicto no solo entre el giro pragmatista contemporáneo de Quine y Kuhn, y el compromiso sustantivo con la noción de método, sino que también plantea una tensión en la posición pragmatista de Hook: entre su compromiso cientificista y el legado "experimentalista" de Dewey.

En cambio, considero que la crítica de Rorty contra Dewey resulta más débil, no solo porque, como se le ha reprochado, su lectura del clásico se vuelve en ocasiones poco caritativa o, incluso, deficiente, ${ }^{58}$ sino, principalmente, debido a la deuda que mantiene para con el clásico. Cuando denuncia, por ejemplo, una tensión entre la dimensión descriptiva y la dimensión normativa o prescriptiva de la propuesta pragmatista de Dewey, Rorty no solo corre el riesgo de ser injusto en calidad de intérprete, sino que en

57 Aunque se encuentre meramente sugerido, mi lectura es compatible con la presentación posterior del pragmatismo rortyano respecto del antiautoritarismo; cf. Richard Rorty, El pragmatismo, una versión: Antiautoritarismo en epistemología y ética (Barcelona: Ariel, 2000), 7-48; Rorty, Philosophy as Cultural Politics, 3-9; Robert Brandom, "Achieving the Enlightenment", en A Version: Anti-Authoritarianism in Epistemology and Ethics, de Richard Rorty, ed. por Eduardo Mendieta (Cambridge: Harvard University Press, 2021).

58 Voparil, “Rorty and Dewey Revisited”, 375-380; Hildebrand, “Dewey, Rorty, and Brandom”, 99-130. 
virtud de sus compromisos filosóficos con el clásico genera una tensión en su propia posición, pues, como explicita por caso en su crítica a Hook, el neopragmatista adhiere al "experimentalismo" ético del clásico, concede a Dewey y adhiere en particular a la tesis del continuo, medios-fines de acuerdo con lo cual los medios-fines, hechos y valores se evalúan conjuntamente en el contexto particular de investigación. ${ }^{59}$

Tampoco resulta satisfactoria, a mi juicio, la objeción de Rorty que plantea un dilema a la posición de Dewey acerca del método, ya que, como se ha señalado, cabe rechazar que haya cánones o principios universales de corrección y justificación del conocimiento científico (aplicables a todo tiempo y lugar), pero de ello no se sigue que no hay método en absoluto y que "vale todo" en la práctica de la investigación científica, posición que Feyerabend llamó "anarquismo metodológico". 60

En el caso de la propuesta de Dewey, su comprensión general de la investigación y su defensa de una posición contextualista no resultan alternativas exclusivas y excluyentes. De manera que Rorty construye, a mi juicio, un falso dilema al plantear las opciones disponibles a Dewey acerca de cómo entender el

59 Elizabeth Anderson, “Dewey's Moral Philosophy”, Stanford University, acceso el 7 de abril de 2021, https://plato. stanford.edu/archives/win2019/entries/dewey-moral/a period of extraordinary social, economic, demographic, political andtechnological change. During his lifetime the United States changedfrom a rural to an urban society, from an agricultural to anindustrial economy, from a regional to a world power. It absorbedmillions of immigrants from Europe and Asia, but faced wrenchingconflicts between capital and labor as they were integrated into theurban industrial economy. As the face-to-face communal life of smallvillages and towns waned, it confronted the need to create new formsof community life capable of sustaining democracy on urban andnational scales. Dewey believed that neither traditional moral normsnor traditional philosophical ethics were able to cope with theproblems raised by these dramatic transformations. Traditionalmorality was adapted to conditions that no longer existed. Hideboundand unreflective, it was incapable of changing to address the problemsraised by new circumstances. Traditional philosophical ethics soughtto discover and justify fixed moral goals and principles by dogmaticmethods. Its preoccupation with reducing the diverse sources of moralinsight to a single fixed principle subordinated practical service toordinary people to the futile search for certainty, stability, andsimplicity. In practice, both traditional morality and philosophicalethics served the interests of elites at the expense of mostpeople. To address the problems raised by social change, moralpractice needed to acquire the disposition to respond intelligently tonew circumstances. Dewey saw his reconstruction of philosophicalethics as a means to effect this practical reconstruction., Dewey's ethics replaces the goal of identifying an ultimate endor supreme ethical principle with the goal of identifying a method forimproving our value judgments. Dewey argued that ethical inquiry isthe use of reflective intelligence to revise our judgments in light ofthe consequences of acting on them. Value judgments are tools forsatisfactorily redirecting conduct when habits fail. As tools, theycan be evaluated instrumentally. We test our value judgments byputting them into practice and seeing whether the results aresatisfactory - whether they solve our problems with acceptableside-effects, whether they enable successful responses to novelproblems, whether living in accordance with alternative valuejudgments yields more satisfactory results. We make moral progress byadopting habits of reflectively revising our value judgments inresponse to the widest consequences for everyone of following them. The conditions of warrant for value judgments lie in human conduct,not in any a priori fixed reference point outside of conduct,such as God's commands, Platonic Forms, pure reason, ornature. Dewey offers a naturalistic metaethic of value judgments,grounded in developmental and social psychology.'”'container-title":"The Stanford Encyclopedia of Philosophy','edition":"Winter 2019'”'"publisher":"Metaphysics Research Lab, Stanford University','source":"Stanford Encyclopedia of Philosophy"',title":"Dewey's Moral Philosophy"',URL":"https://plato.stanford.edu/archives/win2019/

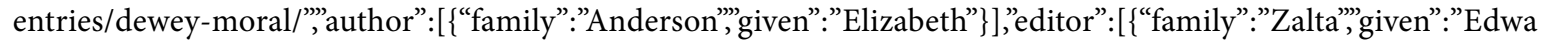
rd N.”\}],"accessed”:\{“date-parts”:[[“2020”,11,28]]\},"issued”:\{“date-parts”:[[“2019”]]\}\}\}],"schema”:”https://github.com/ citation-style-language/schema/raw/master/csl-citation.json"\}

60 Alan Chalmers, What Is This Thing Called Science? (Cambridge: Hackett Publishing, 2013), 138-49; Ricardo Gómez, La dimensión valorativa de las ciencias (Bernal: Universidad Nacional de Quilmes, 2014), 93-95. 
método experimental respecto de una disyunción excluyente: o bien respecto de la práctica social o bien respecto de una técnica específica.

La teoría de investigación de Dewey no aspira a establecer una definición esencialista del conocimiento ni una concepción universalista del método; en cambio, pretende ofrecer una comprensión general de la práctica de investigación a partir del estudio de casos paradigmáticamente satisfactorios (de investigaciones consideradas exitosas). ${ }^{61}$ La propuesta deweyana, pues, no adolece de generalidad, dado que se refiere a un caso potencialmente infinito de investigaciones, ni tampoco deja de reconocer que cada caso concreto de investigación tiene lugar en y se dirige a resolver una situación problemática particular.

La propuesta de Dewey así presentada, además, no es incompatible con los lineamientos que traza Rorty acerca de su propia posición. En otros textos, en efecto, reconoce su deuda con el clásico para su comprensión antidualista de la investigación como estrategia de recontextualización. ${ }^{62}$ Cabe preguntar, pues, si la preferencia rortyana por el anarquismo de Feyerabend en vez del pluralismo de Kuhn y cuánto de su desacuerdo con la posición metodológica del clásico no se limita a más que una cuestión de énfasis retórico.

\section{Metodología}

Dewey enfatiza a lo largo de su obra el aspecto metodológico de su propuesta hasta el punto de que llega a sostener que la contribución de su concepción experimental de la experiencia consiste en ofrecer un método para la filosofía, que manda, para averiguar qué significa "subjetivo", "objetivo", "físico", "mental", o cualquier otro término filosófico, ir a la experiencia y observar cómo operan y para qué se instituyen los conceptos en cuestión. ${ }^{63}$

\section{Exposición}

En primer lugar, en la propuesta deweyana, el método consiste en el "punto de partida práctico” ${ }^{64} \mathrm{La}$

61 Dewey, La miseria de la epistemología, 113-116; Dewey, "Experience, Knowledge and Value”, 533.

62 Rorty, "Method, Social Science and Social Hope"; Rorty, Objectivity, Relativism and Truth, 93-112.

63 John Dewey, Essays in experimental logic (Nueva York: Dover Publications, 1954), 38-46; Dewey, The Influence of Darwin on Philosophy, 238-239; Dewey, Experience and Nature, 8-9, 22-23. Cabe notar que, así presentada, la propuesta metodológica de Dewey resulta compatible en general con la perspectiva wittgensteiniana de Rorty; véase Rorty, Philosophy as Cultural Politics, 3-9, 160-75; Colin Koopman, "Rorty's Linguistic Turn: Why (More Than) Language Matters to Philosophy", Contemporary Pragmatism 8, n. 1 (2011): 62-63; Susan Dieleman, "Locating Rorty: Feminism and Poststructuralism, Experience and Language", The Pluralist 9, n. 3 (2014), 116-17.

64 Hildebrand, "John Dewey”; Gregory Pappas, “The Starting Point of Dewey's Ethics and Sociopolitical Philosophy”, en The Oxford Handbook of Dewey, ed. por Steven Fesmire (Oxford University Press, 2019), 234-254.along with Charles Sanders Peirce and William James,and arguably the most prominent American intellectual for the firsthalf of the twentieth century. Dewey's educational theories andexperiments had a global reach, his psychological theories had asizable influence in that growing science, and his writings aboutdemocratic theory and practice deeply influenced debates in academicand practical quarters for decades. In addition, Dewey developedextensive and often systematic views in ethics, epistemology, logic,metaphysics, aesthetics, and philosophy of religion. Because Deweytypically 
reflexión filosófica, y teórica en general, debe partir de la "experiencia primaria", tal como es vivida, para recoger los primeros datos y establecer el problema en cuestión. ${ }^{65}$ Dewey enfatiza así el carácter eminentemente práctico del contexto en que tiene lugar la actividad de conocimiento. ${ }^{6}{ }^{7}$ Toda investigación tiene lugar en y es motivada por una situación particular de conflicto en que se encuentran en tensión, por ejemplo, distintos hábitos de conducta disponibles o prácticas sociales establecidas, y resulta indeterminado cómo actuar adecuadamente. La identificación del problema, pues, debe ser testeada en la investigación y su establecimiento constituye el primer paso y avance hacia su resolución. ${ }^{67}$

En segundo lugar, Dewey mantiene que, además de ser el punto de partida, la experiencia debe ser también el "punto terminal" de la investigación; desafortunadamente, la formulación de este segundo precepto ha dado lugar a confusión. El énfasis sobre la función de "tribunal" de la experiencia en el requisito de testear la corrección de los productos de la reflexión, junto con otros pasajes sobre la caracterización del

took a genealogical approach that couched his own viewwithin the larger history of philosophy, one may also find a fullydeveloped metaphilosophy in his work., Dewey's pragmatism—or, "cultural naturalism",which he favored over "pragmatism" and"instrumentalism"- may be understood as a critiqueand reconstruction of philosophy within the larger ambit of aDarwinian worldview (Lamont 1961; MW4: 3

65 Dewey, Experience and Nature, 3-5, 36.

66 Cabe advertir que, aunque Dewey caracteriza la experiencia "primaria” como "no cognitiva”, "no reflexiva” o, incluso, no discursiva, no excluye con ello todo elemento reflexivo e intelectual, sino que se refiere a experiencias en que el conocimiento no es la actividad principal; cf. Bernstein, Filosofía y democracia, 72. La experiencia "cualitativa", “inmediata" que se tiene, es eminentemente práctica; cf. Ángel Faerna, "In Search of Lost Body: On Pragmatism, Experience and Language", The Journal of the Central-European Pragmatism Forum 9, n. 2 (2018), 111. El reparo en el uso de "práctica/o" para referirse a la experiencia primaria "tal como es vivida" se debe al prejuicio cultural que pesa sobre la expresión que impone una comprensión estrecha y sesgada: cf. Pappas, "The Starting Point”, 237-238.we must understand how his approach in these areas of philosophy was a consequence of what he thought should be the starting point of philosophy, that is, his metaphilosophy. Dewey prescribed that philosophers should make an effort to be "empirical" and to take "experience" seriously, but these claims are subject to misunderstandings. The first section of this chapter clarifies what they mean. The second section considers the difference that Dewey's form of empirical philosophy makes in ethics and third section the difference it makes in approaching sociopolitical problems. Dewey proposes a much more radical approach than similar contemporary approaches that are interested in a shift from traditional approaches centered on ideal theories and abstractions toward a more nonideal contextualist, problem-centered, and inquiry-oriented approach.'”container-title":"The Oxford Handbook of Dewey"',ISBN":"978-0-19-0491192'”'language":"en'”'note":"DOI: 10.1093/oxfordhb/9780190491192.013.7',"page":"234-254,"'publisher":"Oxford University Press'”'source":"DOI.org (Crossref

67 Dewey, La miseria de la epistemología, 120-121. Sobre la importancia de no partir de diagnósticos previos y establecer el problema en la investigación, véase Pappas, “The Starting Point of Dewey's ethics”.we must understand how his approach in these areas of philosophy was a consequence of what he thought should be the starting point of philosophy, that is, his metaphilosophy. Dewey prescribed that philosophers should make an effort to be "empirical" and to take "experience" seriously, but these claims are subject to misunderstandings. The first section of this chapter clarifies what they mean. The second section considers the difference that Dewey's form of empirical philosophy makes in ethics and third section the difference it makes in approaching sociopolitical problems. Dewey proposes a much more radical approach than similar contemporary approaches that are interested in a shift from traditional approaches centered on ideal theories and abstractions toward a more nonideal contextualist, problem-centered, and inquiry-oriented approach.,'”container-title":"The Oxford Handbook of Dewey"',ISBN":"978-0-19-049119-2",'language":"en'”'note":"DOI: 10.1093/oxfordhb/9780190491 192.013.7"',page":"234-254"',publisher":"Oxford University Press"',source":"DOI.org (Crossref 
concepto por parte del clásico, motivaron una mala comprensión de la propuesta pragmatista respecto de una posición fundacionista. ${ }^{68}$

En efecto, la experimentación se encuentra en el corazón de la comprensión de Dewey de la investigación, pero, al mismo tiempo que destaca el estatus hipotético y revisable en función de la evidencia de los juicios prácticos producto de la investigación, insiste también en su carácter creativo, ${ }^{69}$ ya que el fin de la investigación es establecer cómo actuar en una situación particular en que las prácticas y los hábitos disponibles se encuentran en conflicto. Y para establecer un nuevo modo de conducta adecuado a la situación inédita en cuestión resulta crucial considerar y proyectar en la imaginación posibles cursos de acción en función del desarrollo esperado de la situación a la que conducen. Además, en los casos paradigmáticos de deliberación moral, se encuentran indeterminados no solo los medios de acción (cómo actuar para alcanzar un fin preestablecido), sino también el fin mismo. Se debe establecer en la evaluación cuál es el fin, valor o bien de la situación en cuestión (como vimos, fines y medios se evalúan

68 Richard Shusterman, “Dewey on Experience: Foundation or Reconstruction?”, The Philosophical Forum 26, n. 2 (1994): 127-48; Gregory Pappas, John Dewey’s Ethics: Democracy as Experience (Indiana University Press, 2008), 24; Colin Koopman, "Conduct Pragmatism: Pressing Beyond Experientialism and Lingualism”, European Journal of Pragmatism and American Philosophy 6, n. ${ }^{\circ} 2$ (2014): 150-152.

69 Anderson, "Dewey's Moral Philosophy”.a period of extraordinary social, economic, demographic, political andtechnological change. During his lifetime the United States changedfrom a rural to an urban society, from an agricultural to anindustrial economy, from a regional to a world power. It absorbedmillions of immigrants from Europe and Asia, but faced wrenchingconflicts between capital and labor as they were integrated into theurban industrial economy. As the face-to-face communal life of smallvillages and towns waned, it confronted the need to create new formsof community life capable of sustaining democracy on urban andnational scales. Dewey believed that neither traditional moral normsnor traditional philosophical ethics were able to cope with theproblems raised by these dramatic transformations. Traditionalmorality was adapted to conditions that no longer existed. Hideboundand unreflective, it was incapable of changing to address the problemsraised by new circumstances. Traditional philosophical ethics soughtto discover and justify fixed moral goals and principles by dogmaticmethods. Its preoccupation with reducing the diverse sources of moralinsight to a single fixed principle subordinated practical service toordinary people to the futile search for certainty, stability, andsimplicity. In practice, both traditional morality and philosophicalethics served the interests of elites at the expense of mostpeople. To address the problems raised by social change, moralpractice needed to acquire the disposition to respond intelligently tonew circumstances. Dewey saw his reconstruction of philosophicalethics as a means to effect this practical reconstruction., Dewey's ethics replaces the goal of identifying an ultimate endor supreme ethical principle with the goal of identifying a method forimproving our value judgments. Dewey argued that ethical inquiry isthe use of reflective intelligence to revise our judgments in light ofthe consequences of acting on them. Value judgments are tools forsatisfactorily redirecting conduct when habits fail. As tools, theycan be evaluated instrumentally. We test our value judgments byputting them into practice and seeing whether the results aresatisfactory - whether they solve our problems with acceptableside-effects, whether they enable successful responses to novelproblems, whether living in accordance with alternative valuejudgments yields more satisfactory results. We make moral progress byadopting habits of reflectively revising our value judgments inresponse to the widest consequences for everyone of following them. The conditions of warrant for value judgments lie in human conduct,not in any a priori fixed reference point outside of conduct,such as God's commands, Platonic Forms, pure reason, ornature. Dewey offers a naturalistic metaethic of value judgments,grounded in developmental and social psychology.,'”container-title":"'The Stanford Encyclopedia of Philosophy"',edition":"Winter 2019'”'publisher":"Metaphysics Research Lab, Stanford University"','source":"Stanford Encyclopedia of Philosophy'"'title":"Dewey's Moral Philosophy'”'URL":"https://plato.stanford.edu/archives/win2019/

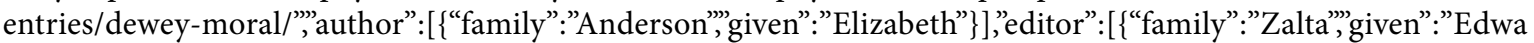
rd N.”\}],”accessed”:\{“date-parts”:[[“2020”,11,28]]\},"issued”:\{“date-parts”:[[“2019”]]\}\}\}],"schema”:"https://github.com/ citation-style-language/schema/raw/master/csl-citation.json”\} 
y determinan recíprocamente). Dewey llega a comprender, entonces, la deliberación respecto de un "ensayo imaginario" ${ }^{70}$ De manera que bien podría caracterizarse el segundo momento de la metodología pragmatista como el ejercicio de la imaginación experimental.

El punto central de la propuesta de Dewey para la comprensión de la investigación no se encuentra, entonces, a mi juicio, en la confrontación o contrastación de los productos de la reflexión con la experiencia desnuda, sino en el movimiento cíclico de ida y vuelta, o retroalimentación entre teoría y práctica, imaginación y experimentación. ${ }^{71}$ Más que expresar la exigencia epistemológica de justificación o fundamentación empírica de los productos de la investigación, la insistencia de Dewey de "volver a la experiencia" se comprende mejor, a mi juicio, como la advertencia de no perder de vista el contexto práctico social sobre el que se anclan y del que dependen la investigación y los productos de la reflexión.

\section{Aplicación}

Se han identificado y distinguido, por tanto, tres funciones de la "experiencia como método" en la propuesta metafilosófica pragmatista de Dewey ${ }^{72}$ : a) crítica de los presupuestos teóricos dualistas de la tradición, b) denuncia de errores comunes en la investigación filosófica y c) reconstrucción de la filosofía. Como observamos, el análisis de la experiencia de Dewey conduce a un diagnóstico teórico (históricogenealógico) de la "industria epistemológica" y los "puzles" metafísicos y epistemológicos a los que da lugar (a), mientras que (b) adopta la forma típica de la denuncia de la "falacia filosófica”. Además, la crítica de la tradición mediante la aplicación del método se encontraba articulada con (c) que involucraba no solo una recuperación de la tarea de la filosofía, sino también la reconstrucción entera de áreas de la disciplina y sus conceptos centrales. ${ }^{73}$ Como las funciones (a) y (b) han sido ilustradas indirectamente en la discusión anterior, a continuación, sugeriré cómo la propuesta pragmatista puede realizar una contribución positiva para el estudio de injusticias epistémicas.

El término ha sido acuñado por Miranda Fricker para referirse a una forma de discriminación que afecta a las personas en calidad de sujetos de conocimiento. Las injusticias testimoniales consisten en un "déficit prejuicioso de credibilidad" que se produce cuando un prejuicio social identitario afecta a un oyente de manera que le otorga menor credibilidad al hablante de la que le atribuiría a otra persona no percibida como miembro del grupo social en cuestión en condiciones semejantes. ${ }^{74}$

70 En el original "dramatic rehearsal"; cf. John Dewey, Teoría de la valoración: Un debate con el positivismo sobre la dicotomía de hechos y valores, ed. por Ángel Faerna y María Aurelia Di Berardino (Madrid: Biblioteca Nueva, 2008), 100-128, 151.

71 Anderson, "Dewey's Moral Philosophy"; Pappas, John Dewey's Ethics."plainCitation”:"Anderson, «Dewey's Moral Philosophy»; Pappas, John Dewey’s Ethics: Democracy as Experience.","noteIndex”:70\},"citationItems”:[\{“id”:318,"u ris":[“http://zotero.org/users/local/AFtThozY/items/DNAZSLHM”],"uri”:[“http://zotero.org/users/local/AFtThozY/ items/DNAZSLHM”],"itemData”:\{“id”:318,"type”:"entry-encyclopedia”'”abstract”:”John Dewey (1859-1952

72 Pappas, “John Dewey's Radical Logic: The Function of the Qualitative in Thinking”; Hildebrand, "John Dewey”.

73 John Dewey, "Context and Thought", University of California Publications in Philosophy 12, n. 3 (1931): 19; Pappas, "What Difference Can "Experience" Make to Pragmatism?", 202.

74 En el ejemplo central tomado de Matar a un ruiseñor, al no creerle a Tom Robinson por ser negro, el jurado falla en su deber epistémico; cf. Miranda Fricker, Epistemic Injustice: Power and the Ethics of Knowing (Oxford University Press, 2007), 69:21-22, 27. 
Sullivan ha defendido la epistemología pragmatista para el análisis de las injusticias epistémicas, ya que considera que brinda herramientas más efectivas para combatir las injusticias testimoniales. ${ }^{75}$ En efecto, la razón por la que las injusticias testimoniales son tan persistentes es porque son causadas por prejuicios sociales que resultan invisibles para quienes se ven afectados en su juicio perceptivo por ellos, pues los prejuicios son generalizaciones falsas cuya creencia resulta resistente a la evidencia. ${ }^{76}$ La concepción tradicional de corte individualista del conocimiento como representación adecuada, valorativamente neutral, de los hechos resulta deficiente para poder comprender cómo operan los prejuicios y cómo contrarrestar su efecto nocivo. En cambio, desde la perspectiva pragmatista, aunque quienes encuentran comprometida su percepción no pueden observar personalmente el efecto epistémico negativo en cuestión, sus juicios perceptivos sesgados se encuentran sujetos a corrección por parte de sus pares no afectados en su interacción con los demás miembros de la comunidad. ${ }^{77} \mathrm{~A}$ su vez, ofrece una alternativa a la neutralidad axiológica: los fines y valores deben evaluarse en función de cómo impactan la investigación: si inclinan la evaluación de la evidencia y la investigación hacia una meta preestablecida o permiten revelar nueva evidencia. ${ }^{78} \mathrm{El}$ criterio general, sugiero, podría aplicarse a los prejuicios para distinguirlos de los estereotipos positivos para poder diferenciar estereotipos sociales positivos de prejuicios. ${ }^{79}$

\section{Conclusiones}

Del abordaje crítico de la cuestión desarrollado en la primera sección se desprende una defensa de la propuesta metodológica pragmatista ante la crítica interna de Rorty. La tesis principal defendida mantiene que la comprensión general de la investigación, como la que despliega Dewey, y en ocasiones retomada por Rorty, ofrece una propuesta metodológica pragmatista que no se compromete con una comprensión universalista del método ni tampoco redunda en un "anarquismo", como el que recomienda Rorty.

El examen de la crítica revela que los argumentos del neopragmatista atacan una comprensión universalista del método, que se compromete con la tesis de que pueden establecerse principios epistemológicos acerca del correcto desarrollo de la investigación, que constituyen la "lógica" del conocimiento científico y son aplicables a todo tiempo y todo lugar. De manera tal que el argumento neopragmatista resulta efectivo contra la posición de Hook (presuntamente inspirada en el empirismo lógico), pero no brinda razones de peso para preferir el abandono total de la noción de método por sobre una versión debilitada de este ni adherir a la tesis anarquista en vez de una posición pluralista.

75 Shannon Sullivan, "On the Harms of Epistemic Injustice: Pragmatism and Transactional Epistemology", en The Routledge Handbook of epistemic Injustice, ed. por José Medina, Ian Kidd y Gaile Pohlhaus (Londres: Routledge, 2017), 205, 2010-2211.

76 Fricker, Epistemic Injustice: Power and the Ethics of Knowing, 69:35.

77 Inspirado, además, en Wittgenstein, Medina enfatiza el rol de la "fricción epistémica" en José Medina, The Epistemology of Resistance: Gender and Racial Oppression, Epistemic Injustice, and Resistant Imaginations, Studies in Feminist Philosophy (Oxford: Oxford University Press, 2013).

78 Elizabeth Anderson, "Uses of Value Judgments in Science: A General Argument, with Lessons from a Case Study of Feminist Research on Divorce", Hypatia 19, n. ${ }^{\circ} 1$ (2004): 1-24.

79 Fricker, Epistemic injustice, 31-32. 
Como el propio Rorty sostiene, el pragmatismo puede mantener su compromiso con una perspectiva naturalista sin ser cientificista, pues ofrece una comprensión general de la investigación respecto de la práctica social autocorrectiva: los cánones, las normas o los principios del "método" que rigen la investigación dependen del consenso de la comunidad en el contexto particular. Esto es, el consenso entre los investigadores, especialistas en un área, acerca de cuáles son las mejores teorías, las estrategias metodológicas más productivas, la mejor evidencia disponible, etc.; consenso que se encuentra sujeto a revisión continua por parte de la misma comunidad ante los cambios que ocurran. De manera que, aunque no haya un único método ahistórico y universal, la práctica de investigación dispone de criterios de corrección y puede evaluar métodos mejores o peores, propuestas más o menos satisfactorias.

La exposición presentada en la segunda sección se enfoca en el aspecto metafilosófico de la propuesta pragmatista y destaca entre sus principales contribuciones el contextualismo pluralista y el esquema de feedback loop entre teoría y observación en la imaginación experimental.

El apartado "Aplicación" indica, por un lado, cómo Dewey aplicó su propuesta metodológica para la crítica de la tradición en su lectura de la historia de la filosofía, pero, además, se defiende la potencial fecundidad de la propuesta para la discusión filosófica actual acerca de las injusticias epistémicas. Y por otro, se identifican aportes pragmatistas en trabajos recientes en el área (como los casos de Anderson, Dieleman o Sullivan, por mencionar algunos de los autores destacados), aunque las observaciones no pueden dejar de ser esquemáticas y generales dadas las limitaciones, además de las potenciales ventajas de la perspectiva pragmatista de la investigación por sobre la epistemología ortodoxa para contribuir a la comprensión de cómo operan los prejuicios en las injusticias testimoniales.

\section{Bibliografía}

Anderson, Elizabeth. "Dewey’s Moral Philosophy”. Stanford University. Acceso el 7 de abril de 2021. https://plato. stanford.edu/archives/win2019/entries/dewey-moral/.

Anderson, Elizabeth. "Uses of Value Judgments in Science: A General Argument, with Lessons from a Case Study of Feminist Research on Divorce". Hypatia 19, n. 1 (2004): 1-24.

Bernstein, Richard. Filosofía y democracia: John Dewey. Ed. por Ramón del Castillo. Barcelona: Herder, 2011.

Bernstein, Richard J. The Pragmatic Turn. Malden: Polity Press, 2013.

Brandom, Robert. "Achieving the Enlightenment". En A Version: Anti-Authoritarianism in Epistemology and Ethics, de Richard Rorty, editado por Eduardo Mendieta. Cambridge: Harvard University Press, s. f.

Brandom, Robert. Perspectives on Pragmatism: Classical, Recent, and Contemporary. Harvard: Harvard University Press, 2011.

Brown, Harold. “Filosofía de la ciencia del empirismo lógico”. En La nueva filosofía de la ciencia, 17-102. Madrid: Tecnos, 1983.

Chalmers, Alan. What is this Thing Called Science? Cambridge: Hackett Publishing, 2013.

Dewey, John. "Context and Thought". University of California Publications in Philosophy 12, n. 3 (1931): 203ff.

Dewey, John. "El concepto de arco reflejo en psicología". En La miseria de la epistemología, editado por Ángel Faerna. Madrid: Biblioteca Nueva, 2000.

Dewey, John. Essays in Experimental Logic. Nueva York: Dover Publications, 1954.

Dewey, John. Experience and Nature. Nueva York: Dover Publications, 1958.

Dewey, John. "Experience, Knowledge, and Value: A Rejoinder". En The Philosophy of John Dewey, editado por Paul Schilpp y Hahn Lewis, 517-608. Chicago: Open Court, 1989.

Dewey, John. La busca de la certeza. México: Fondo de Cultura Económica, 1952. 
Dewey, John. Teoría de la valoración: Un debate con el positivismo sobre la dicotomía de hechos y valores. Ed. por Ángel Faerna y María Aurelia Di Berardino. Madrid: Biblioteca Nueva, 2008.

Dewey, John. The Influence of Darwin on Philosophy and Other Essays. Nueva York: Prometheus Books, 1997.

Dewey, John. The Philosophy of John Dewey. Chicago: Open Court, 1989.

Dewey, John y Sidney Ratner. The Later Works of John Dewey. Vol. 6, 1925-1953: 1931-1932, Essays, Reviews, and Miscellany. Carbondale: Southern Illinois University Press, 1985.

Dieleman, Susan. "Locating Rorty: Feminism and Poststructuralism, Experience and Language". The Pluralist 9, n. ${ }^{\circ}$ 3 (2014): 110-20.

Faerna, Ángel. "In Search of Lost Body: On Pragmatism, Experience and Language". The Journal of the CentralEuropean Pragmatism Forum 9, n. 2 (2018): 107-118.

Faerna, Ángel. Introducción a la teoría pragmatista del conocimiento. Madrid: Siglo XXI, 1996.

Faerna, Ángel. "Pragmatismo conceptualista: La teoría del conocimiento de C. I. Lewis". Tesis doctoral. Universidad Complutense de Madrid, 2002. Acceso el 7 de abril de 2021. https://eprints.ucm.es/id/eprint/2310/.

Feyerabend, Paul. Contra el método: Esquema de una teoría anarquista del conocimiento. Buenos Aires: Hyspamerica, 1984.

Fricker, Miranda. Epistemic Injustice: Power and the Ethics of Knowing. Oxford: Oxford University Press, 2007.

Gómez, Ricardo. La dimensión valorativa de las ciencias: Hacia una filosofía política. Bernal: Universidad Nacional de Quilmes, 2014.

Hempel, Carl. Filosofía de la ciencia natural. Madrid: Alianza, 1984.

Hempel, Carl . "Problemas y cambios en el criterio empirista de significado". En El positivismo lógico, editado por Alfred Ayer, 115-36. Madrid: Fondo de Cultura Económica, 1978.

Hildebrand, David. "Dewey, Rorty, and Brandom: The Challenges of Linguistic Neopragmatism". En The Oxford Handbook of Dewey, de David Hildebrand, editado por Steven Fesmire, 98-130. Oxford: Oxford University Press, 2019. doi:10.1093/oxfordhb/9780190491192.013.5.

Hildebrand, David . "John Dewey". Stanford University. Acceso el 7 de abril de 2021. https://plato.stanford.edu/ archives/win2018/entries/dewey/.

Hildebrand, David. "Introduction". European Journal of Pragmatism and American Philosophy 6, n. ${ }^{\circ}$ (2014): 6-13. https://doi.org/10.4000/ejpap.275.

Hook, Sidney. The Quest for Being. Nueva York: Macmillan, 1961.

Johnson, Mark. "Experiencing Language: What's Missing in Linguistic Pragmatism?". European Journal of Pragmatism and American Philosophy 6, n. ${ }^{\circ} 2$ (2014): 14-27. https://doi.org/10.4000/ejpap.284.

Klimovsky, Gregorio. Las desventuras del conocimiento científico: Una introducción la epistemología. Buenos Aires: A-Z, 1997.

Koopman, Colin. "Conduct Pragmatism: Pressing Beyond Experientialism and Lingualism". European Journal of Pragmatism and American Philosophy 6, n. ${ }^{\circ} 2$ (2014): 145-174. https://doi.org/10.4000/ejpap.313.

Koopman, Colin. "Language Is a Form of Experience: Reconciling Classical Pragmatism and Neopragmatism". Transactions of the Charles S. Peirce Society 43, n. ${ }^{\circ} 4$ (2007): 694-727.

Koopman, Colin. "Rorty's Linguistic Turn: Why (More Than) Language Matters to Philosophy". Contemporary Pragmatism 8, n. ${ }^{\circ} 1$ (2011): 61-84. doi:10.1163/18758185-90000183.

Kuhn, Thomas. La estructura de las revoluciones científicas. Buenos Aires: Fondo de Cultura Económica, 1988.

Levine, Steven. Pragmatism, Objectivity, and Experience. Cambridge: Cambridge University Press, 2019.

Marcos, Alfredo. Ciencia y acción: Una filosofía práctica de la ciencia. México: Fondo de Cultura Económica, 2010.

Medina, José. The Epistemology of Resistance: Gender and Racial Oppression, Epistemic Injustice, and Resistant Imaginations. Oxford: Oxford University Press, 2013.

Pappas, Gregory. John Dewey's Ethics: Democracy as Experience. Bloomington: Indiana University Press, 2008.

Pappas, Gregory. "John Dewey's Radical Logic: The Function of the Qualitative in Thinking”. Transactions of the Charles S. Peirce Society 52, n. ${ }^{\circ} 3$ (2016): 435-468. https://doi.org/10.2979/trancharpeirsoc.52.3.08.

Pappas, Gregory. "The Starting Point of Dewey's Ethics and Sociopolitical Philosophy". En The Oxford Handbook of Dewey, editado por Steven Fesmire, 234-54. Oxford: Oxford University Press, 2019. doi:10.1093/ oxfordhb/9780190491192.013.7.

Pappas, Gregory. “What Difference Can 'Experience' Make to Pragmatism?”. European Journal of Pragmatism and American Philosophy 6, n. ${ }^{\circ} 2$ (2014): 200-227. https://doi.org/10.4000/ejpap.322. 
Popper, Karl. La lógica de la investigación científica. Madrid: Tecnos, 1980.

Reichenbach, Hans. Experience and Prediction. Chicago: University of Chicago Press, 1938.

Rorty, Richard. "Dewey's Metaphysics". En Consequences of Pragmatism, 72-89. Minneapolis: University of Minnesota Press, 1982.

Rorty, Richard. El pragmatismo, una versión: Antiautoritarismo en epistemología y ética. Barcelona: Ariel, 2000.

Rorty, Richard. "Introduction". En The Later Works of John Dewey. Vol. 8, 1925-1953: 1933. Essays and How We Think, editado por Jo Ann Boydston, IX-XVIII. Carbondale: Southern Illinois University Press, 1986.

Rorty, Richard. "Method, Social Science and Social Hope”. En Consequences of Pragmatism, 1912-210. Minneapolis: University of Minnesota Press, 1982.

Rorty, Richard. Objectivity, Relativism, and Truth. Cambridge: Cambridge University Press, 1991.

Rorty, Richard. Philosophy as Cultural Politics: Philosophical Papers. Cambridge: Cambridge University Press, 2007.

Rorty, Richard. "Pragmatism Without Method". En Philosophical Papers. Vol. 1: Objectivity, Relativism, and Truth, 63-77. Cambridge: Cambridge University Press, 1991. doi:10.1017/CBO9781139173643.005.

Rorty, Richard. "Response To James Gouinlock”. En Rorty \& Pragmatism: The Philosopher Responds to His Critics, editado por Herman J. Saatkamp. Nashville: Vanderbilt University Press, 1995.

Shusterman, Richard. “Dewey on Experience: Foundation or Reconstruction?”. The Philosophical Forum 26, n. 2 (1994): 127-48.

Sullivan, Shannon. "On the Harms of Epistemic Injustice: Pragmatism and Transactional Epistemology". En The Routledge Handbook of epistemic Injustice, editado por José Medina, Ian Kidd y Gaile Pohlhaus, 205-2012. Londres: Routledge, 2017.

Voparil, Christopher. "Rorty and Dewey Revisited: Toward a Fruitful Conversation". Transactions of the Charles S. Peirce Society 50, n. 3 (2014): 373-404. https://doi.org/10.2979/trancharpeirsoc.50.3.373.

Wittgenstein, Ludwig. Investigaciones filosóficas. Madrid: Gredos, 2009. 